\title{
Highlights and summary of the 28th annual meeting of the Society for Immunotherapy of Cancer
}

\author{
Paolo A Ascierto ${ }^{1 \dagger}$, David H Munn ${ }^{2 \dagger}$, Anna K Palucka ${ }^{3,4 \dagger}$ and Paul M Sondel ${ }^{5^{*}+}$
}

\begin{abstract}
The 28th annual meeting of the Society for Immunotherapy of Cancer (SITC) was held November 8-10, 2013 in National Harbor MD, and was organized by Paolo Ascierto (Instituto Nazionale Tumori - Fondazione 'G. Pascale'), David Munn (Georgia Regent's University), A. Karolina Palucka (Baylor Institute for Immunology Research) and Paul Sondel (University of Wisconsin). This meeting included over 1100 registered participants from 32 separate countries, making it the largest SITC meeting held to date. It highlighted significant worldwide progress in the development and application of cancer immunology to the practice of clinical oncology, including advances in diagnosis, prognosis and therapy, utilizing a variety of immunological pathways and mechanisms for a variety of oncologic conditions. Presentations and posters demonstrated that many concepts that had been pursued preclinically in the past are now being translated into clinical practice, with clear benefits for patients. One month after this 28th annual meeting, the Journal Science selected the field of Cancer Immunotherapy as the overall \#1 scientific "breakthrough" for 2013.
\end{abstract}

Keywords: Immunotherapy, Cancer, Adaptive immunity, Innate immunity, Adoptive immunotherapy, Immune escape

\section{Introduction}

For over a century, immunology researchers have speculated that the immune mechanisms that are able to provide protection from pathogens, destroy transplanted allogeneic organs, or cause tissue destruction in the form of autoimmune disorders, might be directed selectively against cancer in the form of cancer immunotherapy. While basic research has, for over 60 years, demonstrated the potential for such mechanisms in vitro and in mice, the effective translation into clinical practice has been slower. In 1984, the Society for Immunotherapy of Cancer was established. Its mission is "to improve cancer patient outcomes by advancing the science, development and application of cancer immunology and immunotherapy through our core values of interaction/integration, innovation, translation and leadership in the field." Since then, effective clinical application of immunotherapeutic principles to clinical oncology has

\footnotetext{
* Correspondence: pmsondel@humonc.wisc.edu

${ }^{\dagger}$ Equal contributors

${ }^{5}$ Departments of Pediatrics and Human Oncology, University of Wisconsin, 4159 MACC Fund UW Childhood Cancer Research Wing Wisconsin Institute for Medical Research, 1111 Highland Avenue, Madison, WI 53705, USA Full list of author information is available at the end of the article
}

grown progressively. In 2013, cancer immunotherapy was selected as overall the top field with scientific breakthrough [1]. This year's SITC meeting included a diverse array of effective applications of immune principles into clinical practice, with beneficial impact in the management of many distinct cancers affecting children and adults.

\section{Review}

Keynote presentations

Highlighting this meeting were 2 outstanding keynote presentations regarding cutting- edge strategies that are demonstrating the impact of both innate and T-cell mediated immune effector mechanisms.

\section{Keynote presentation by Giorgio Trinchieri ( $\mathrm{NCl})$}

Humans are a composite of different species including bacterial, fungi, viruses.

Microbial cells outnumber human cells by 10 fold, at genome level in the gut $99 \%$ of genome comes from commensal microbiota. How the inflammatory environment in the host affects immunotherapy and how commensal microbiota affects the response to therapy and to 
immunotherapy are not clear. Skin immunity is regulated by skin microbiota [2]. However, gut commensals have systemic effects and regulate systemic immunity and inflammatory tone.

It is now well documented that inflammation contributes to the development, progression, and treatment of cancer. However, the mechanisms are not yet resolved. It also remains unclear whether commensal bacteria affect inflammation in the sterile tumor microenvironment. Giorgio Trinchieri discussed studies in transplantable murine tumor models that were focused on addressing this issue [3]. It has been found that disruption of the microbiota impairs the response of subcutaneous tumors to CpGoligonucleotide immunotherapy. Similar effects were observed with oxiplatinum or cisplatinum. Oxiplatin but not cisplatin induces immunogenic cell death. These antitumor effects are dependent on the presence of microbiota, even in the absence of immunogenic cell death, suggesting that the immune component may rely on early innate effects rather than adaptive immunity. Mechanistically, in antibiotic-treated or germ-free mice, tumor-infiltrating myeloid-derived cells responded poorly to therapy, resulting in lower cytokine production and tumor necrosis after CpG-oligonucleotide treatment and deficient production of reactive oxygen species and cytotoxicity after chemotherapy. Thus, optimal responses to cancer therapy require an intact commensal microbiota that mediates its effects by modulating myeloid-derived cell functions in the tumor microenvironment [3]. More importantly, the composition of fecal microbiota could be used to segregate mice with high and low intratumoral TNF. This in turn might enable patient stratification in future studies.

\section{Keynote presentation by Carl $\mathrm{H}$. June, recipient of The Richard V. Smalley MD 2013 Award}

Since 2005, SITC honors a luminary in the field who has significantly contributed to the advancement of cancer immunotherapy research by presenting the annual Richard V. Smalley MD Memorial Award, which is associated with the Smalley keynote lecture at the Annual SITC meeting. The awardee this year Carl H. June of the University of Pennsylvania, has led innovative translational research for over 25 years, with the most recent focus being the development of the Chimeric Antigen Receptor modified T-cell (CART) approach. Carl June summarized how the past 15 years of progress have expanded upon the original concept presented by Zelig Eshhar [4], in which variable regions of tumor-reactive monoclonal antibodies (mAbs) $\left(\mathrm{V}_{\mathrm{H}}\right.$ and $\left.\mathrm{V}_{\mathrm{L}}\right)$ are linked to transmembrane and signaling domains of $\mathrm{T}$ cell activating molecules to create membrane based receptors with specificity for the tumor antigen recognized by the original mAb [4]. These receptors can be transfected into T cells, for example with lentiviruses. Preclinical work demonstrated how CD3- $\zeta$ and 41BB signaling components enhanced proliferation and survival of T cells in hypoxic conditions. The initial clinical work has been done with CART reactive to CD-19 on malignant B cells, with progress particularly in chronic lymphocytic leukemia (CLL) in adults and acute lymphoblastic leukemia (ALL) in children [5,6]. As of the SITC meeting, CarlJune's team had treated 35 patients with CLL and 20 with ALL. Of the 20 with ALL, $1 / 2$ had relapsed after allogeneic BMT. Of these 20 children, 17 were in complete remission, and with persistent B cell aplasia; documenting the persistent effects of the CART cells. Toxicities included the persistent B cell aplasia and profound tumor lysis and cytokine storm, seen 1-2 weeks into the treatment for ALL. This cytokine storm has been ameliorated by using anti-IL6 mAb. The B cell aplasia, while undesired, is acceptable, as patients can receive passive replacement of IgG, thus making their B cells "expendable". These CART cells can traffic into the CNS. In ALL patients, it appears that each individual CART cell (or its progeny) can destroy 1000 tumor cells. Ongoing efforts in CarlJune's program, and at other centers, are now moving into analyses of CART reactive with other tumor targets, by using mAbs that recognize antigens expressed on other tumors. Among these are EGFR on glioblastoma, PSMA on prostate cancer, mesothelin on ovarian cancer, HER2 on breast (and other) cancers, and several other targets. Because some of these targets are also expressed on normal tissues that are "not expendable", novel approaches are being developed to decrease the potency or longevity of the CART effect, to decrease potential toxicity. This includes generating "short lived" CART cells by inducing CAR expression with short-lived RNA, rather than transfecting with a DNA construct that remains permanently.

A major question raised for this very exciting technology is the feasibility of wide-scale application. If the efficacy warrants it, wider clinical application should follow. As an example, allogeneic BMT was done at only a handful of centers in the late 1970s; over the past 3 decades hundreds of centers have adopted this complex, yet effective approach, and now over a million BMTs have been performed worldwide. Carl June suggests that high-tech approaches towards cost-effective robotics and automation may become key in enabling the CART approach in order to be expanded broadly and to enable its future application at many sites for large population of patients with cancers that may potentially benefit from this potent immunotherapy.

\section{Overview of sessions}

The 2013 Annual Meeting brought together several broad themes that are timely and relevant for clinical and translational immunotherapy. One theme was activating immunotherapy by administration of either anti-tumor effector cells, antibodies or vaccines. Presentations in this area included sessions on cellular therapeutics ("Adoptive Immunotherapy" session, and the Richard V. Smalley 
lecture), "Vaccines", and on tumor-targeting antibodies ("Antibody Recognition to Engage Cells of the Innate and Adaptive Immune Systems"). A second broad theme within the meeting was tumor-induced negative regulation in the immune system. This was addressed in sessions on "Tumor Immune Resistance and Overcoming It", "Tumor Microenvironment and Innate Cells Recognition" and a session on the immunoregulatory aspects of "Metabolism and Immunity". In recognition of the progress that has been bringing individual agents to the clinic, a Plenary Session was devoted to "Combination Approach in Cancer". Biomarker analysis is becoming increasingly important - both in identifying patients most likely to benefit from therapy, and as markers of response. Sessions were included on "Biomarkers and Immunoscoring" and a Hot-Topic symposium on "Predictive biomarkers in Checkpoint Blockade". Finally, sessions were provided for the update on the development of the Immunoscore, on the Cancer Immunotherapy Trials Network (CITN), and by representatives from the NCI and the FDA.

\section{Tumor microenvironment and innate cells recognition}

Preclinical studies have revealed an unexpected ability of immune system to contribute to the success of chemotherapy and radiotherapy. Anticancer therapies can trigger immune system activation by promoting the release of danger signals from dying tumor cells and/or the elimination of immunosuppressive cells. Two talks discussed the role of myeloid cells in response to chemotherapy. Lionel Apetoh's (INSERM, France) group has recently discovered however that some chemotherapies, such as 5-fluorouracil (5FU) and gemcitabine, exert conflicting effects on anticancer immune responses. Although 5-fluorouracil and Gemcitabine (GEM) selectively eliminated myeloid-derived suppressive cells in tumor-bearing rodents, these chemotherapies promoted the release of IL-1 $\beta$ and the development of proangiogenic IL-17-producing CD4 T cells [7]. Indeed, GEM and 5FU triggers inflammasome in MDSCs and caspase 1 activation in vitro and in vivo leading to IL1 $\beta$ release. The protumor effect of IL1 $\beta$ is via action on the host rather than tumor via impact on CD4+ T cell differentiation and IL17 production driven by MDSCs. Importantly, in a pilot clinical study, two patients with metastatic colorectal cancer received anakinra resulting in reversal of 5FU resistance. Masahisa Jinushi (Hokkaido University) discussed work centered on factors that modulate DAMP-mediated innate immune response. TIM-3 and TIM4 were identified in siRNA-based screen as upregulated in DCs. High TIM-3 expression in tumor associated DCs can be driven by VEGFA and IL10. TIM-3 has multiple effects including suppression of nucleic acid mediated-innate immunity via inhibition of nucleic acid based recognition and type I interferon production. TIM-3 binds HMGB1 and represses endocytosis of nucleic acids in endosomes and moreover DC specific production of TIM3 attenuates anti-tumor effects of chemotherapy [8]. TIM4 promotes lysosmal degradation of tumor antigens in macrophages. TIM4 activates autophagy via interaction with AMPK- $\alpha 1$ after ingestion of apoptotic tumor cells. TIM4 macrophages activated via chemotherapy degrade tumor associated antigens via authophagy and inhibit CD8+ T cells.

Innate immune mechanisms in the tumor microenvironment were further discussed in the talk from Thomas Gajewski's lab (Univeristy of Chicago). There, Xanthenone derivative DMXAA induces STING-dependent and IFN $\alpha$ mediated effects on DCs. This in turn enables inhibition of melanoma via immune effects and induction of memory demonstrated by mice resistance to tumor challenge. As discussed by Anu Wallecha (Advaxis Inc), administration of Listeria-based vaccine leads to induction of high avidity CTLs in a mouse model of prostate cancer. This was associated with reduction of Tregs and IL-10 production in tumors. Also, MDSCs were affected, both monocytic and granulocytic populations, and their suppressive activity was reduced via lower arginase Ievels in this setting.

Tumor microenvironments are complex and myeloid cells are not the only players. Indeed, Peter Nelson (Fred Hutchinson Cancer Research Center) discussed modulating tumor microenvironment responses to genotoxic cancer therapy and impact on fibroblasts via the DNA damage associated secretory response program. These results originated in clinical observations in prostate cancer where 54 patients received prior to surgery 4 cycles mitoxantrone-docetaxel, therapy. No complete clinical responses in tumors were observed even though the DNA damage in cells and stroma could be detected. This is a DNA damage associated secretory program which includes IL6, IL8, and IL33 secretion among others. Similar patterns can be found in breast and ovarian after genotoxic therapy. Molecular analysis revealed alteration of WNT16B in the TME in about $50 \%$ of patients. Mechanistically, $\beta$-catenin targets genes associated with WNT16B post chemo and fibroblasts promote tumor growth via paracrine activation of WNTB16 via NFkB. Clinically, patients without WNT16B induction post chemo had better overall survival.

Lisa Coussens (Oregon Health \& Science University) summed up the session by discussing the leukocyte complexity in human breast cancer. Clinical observations showed that tumor microenvironments dominated by CD68+ and low CD8+ cells (CD68 high/CD8 low phenotype) show poor progression free survival and overall survival across breast cancer subtypes [9]. In a murine model of breast cancer, CSF1/CSF1R blockade leads to a drop in macrophages in primary tumor and an increase in CD8 T cell infiltrate. Macrophage depletion combined with Taxol therapy results in much improved responses in 
terms of tumor regression and inhibition of lung metastasis. Mechanistically, arginase and IL-10 derived from polarized macrophages are inhibited, thereby releasing the brakes on CD8+ T cells. Accordingly, IL-10R blockade results in the inhibition of tumor progression and improved survival in mice which is CD8 T cell dependent, is associated with increased DCs infiltrate and involves IL-12.

\section{Antibody recognition to engage cells of the innate and adaptive immune systems (antibody dependent cell-mediated cytotoxicity: ADCC)}

Certain mAbs and their derivatives can enable tumorselective recognition by their specific interactions with molecules expressed on tumor cell surfaces. Therapeu tically-focused interventions directed to activate immune effector cells, engineer changes in the mAbs, and create fusion proteins of the mAbs and agents that activate effector cells are being developed and used pre-clinically and clinically. The goal is to enable diverse effector cells within the patient that might not otherwise be able to selectively recognize their cancer to become activated and focus their immunologic activities on mediating effective direct or indirect anti-tumor responses.

Raphael Clynes (Columbia University) presented his continuing work on immune activation by antitumor antibodies. In pre-clinical mouse models, his group has demonstrated that effective Fc receptors are involved in at least 2 pathways in the beneficial antitumor effects mediated by tumor reactive mAbs. First, FcRs are essential components in ADCC mediated by cells of the innate immune system, including ADCC by NK cells as well as monocytoid and myeloid elements (Macrophages and neutrophils). Secondly, in immunologically intact animals, FcRs also play a role in enabling uptake of antigen into dendritic cells and effective presentation of them to drive an adaptive immune response. These adaptive responses, driven by tumor reactive antibody, can be further enhanced by elimination of $\mathrm{T}_{\text {reg }}$ cells. Clinical data in patients receiving mAb therapy for HER2+ breast cancer confirm induction of an adaptive immune response following Trastuzumab therapy, with serological evidence of adaptive immune responses to additional antigens, other than HER2, on the patients' cancer [10].

Ron Levy (Stanford University) showed pre-clinical and clinical data demonstrating that the efficacy of tumorreactive $\mathrm{mAbs}$ can be enhanced by co-administration of a second $\mathrm{mAb}$ (anti-CD137, that recognizes 41BB) that further stimulates activated NK cells. Once NK cells are activated (such as via activation through their FcR in the process of initiating ADCC), they upregulate their CD137 expression. At that point, interaction with an agonistic anti-CD137 mAb can further activate the NK cell, resulting in substantial augmentation of ADCC. In vitro studies led to elegant pre-clinical models in immunodeficient mice bearing human lymphoma or breast cancer xenografts. Treatment efficacy with antitumor mAb and human NK cells was substantially augmented when anti-CD137 mAb was co-administered [11]. Ongoing clinical testing of this concept has shown initial exciting results. Some patients that have recurrent lymphoma following Rituximab therapy are showing prolonged responses following combined treatment with Rituximab and anti-CD137. Ron Levy also provided intriguing results looking at beneficial effects of locally injected (i.e., intratumoral) anti-CTLA4 with CpG and anti-OX-40, providing very potent pre-clinical efficacy, with systemic antitumor effects [12]. This suggests that one might "inject locally and treat globally".

Gabrielle Romain (University of Houston) and colleagues have developed a single cell high-throughput microscopy assay for measuring ADCC by anti-CD33 mAbs. Using this approach they have shown that a genetically engineered anti-CD33 mAb (with an Fc-end improved for ADCC) enables more NK cells to mediate ADCC, speeds up the kinetics of conjugation and enables target cell apoptosis with fewer cell-cell contacts.

Beatrix Kotlan (National Institute of Oncology, Budapest, Hungary) and colleagues have been dissecting the specificity of antibodies made by B cells within tumors from 87 patients with melanoma using a sequencing approach of IgG light and heavy chains. Several of these melanoma patients were shown to express a unique GD3 sialated glycoprotein.

Pablo Umana (Roche Glycart AG) described the development and activity of the recently FDA-approved anti-CD20 mAb Gazyva (Obinutuzumab). This is the first drug with "breakthrough therapy designation", and first Fc engineered $\mathrm{mAb}$, to be approved by the FDA. It reacts against an epitope on CD20 that can cause direct mAb-mediated cell death of CD20+ tumor cells, as well as inducing ADCC. Furthermore, the defucosylated $\mathrm{Fc}_{\mathrm{c}}$ end of the mAb enables better interactions with FcRs and enhanced ADCC [13]. In clinical testing, when combined with chlorambucil in the treatment of CLL, Gazyva showed a longer time to progression (26.7 mo. vs. 15.2 mo). Additionally, using genetically engineered mAbs and immunocytokines are in development and testing.

Paul Sondel (University of Wisconsin) summarized preclinical and clinical testing of anti-GD2 mAb for neuroblastoma. An approach tested by the Children's Oncology Group showed that a combination of the chimeric antiGD2 mAb (ch14.18) plus IL2 and GM-CSF to boost ADCC, yielded an increase in event free survival over no immunotherapy (66\% vs. $46 \%$ at 2 years) [14]. Further development includes testing of the humanized form of this mAb linked to IL-2 (the hu14.18-IL2 immunocytokine). Clinical activity was seen in Phase II testing. Furthermore, the involvement of NK cells in the clinical effect is supported by analyses of Killer Immunoglobulin-like 
Receptors (KIR) and their ligands which show greater likelihood of response in patients that have favorable KIR/KIR-Ligand genotypes [15].

\section{Adoptive immunotherapy}

Ex vivo technology is providing the capability to expand, select, and modify varying immune cell types to yield large numbers of antigen specific (tumor specific) cells that can be infused as clinical anti-tumor therapy. Different methodologies are utilizing recognition capabilities of $\mathrm{T}$ cells that are derived from their own TCR repertoire versus conferring specificity by providing them with genetically modified receptors, based on TCR recognition, $\mathrm{mAb}$ recognition, or other ligand-specific receptors. While T cells have been primarily utilized in these trials, these manipulations are also relevant to NK cells, which are also being evaluated. Presentations at SITC focused on the rationale, distinctions and capabilities of these different forms of adoptive cellular immunotherapy and on what clinical settings are showing clinical benefit. This important area of research has made much progress over the past year, as highlighted at this year's meeting by the Richard Smalley Award, going to Carl June, for his and his team's work in pre-clinical and clinical development of chimeric antigen receptor modified T cells (CART) for adoptive immunotherapy (see summary of his lecture, above).

Dirk Busch (Technical University of Munich) and colleagues are investigating the properties of stem cells that can reconstitute immune function. They have shown that the subpopulation of cells that initially respond in a murine immune response are not necessarily the same as those that will expand with the recall response. The ones able to respond upon recall are CD62 high . They can take a single naïve CD62 $2^{\text {high }} \mathrm{T}$ cell, and reconstitute an immunodeficient $\mathrm{RAG}^{-1-}$ mouse with it, and then go through 3 repeat transfers from one $\mathrm{RAG}^{-/-}$recipient to another, each demonstrating successful reconstitution. This demonstrates that central memory $\mathrm{T}$ cells $\left(\mathrm{T}_{\mathrm{CM}}\right)$ are true "adult tissue stem cells", with high regenerative power, and this also makes them good candidates for use with genetically engineered receptors (CARs or TCRs) for cancer therapies.

Stan Riddell (Fred Hutchinson Cancer Research Center) and colleagues have been investigating adoptive immunotherapy pre-clinically and clinically. In adoptive transfer studies in mice they see anti-tumor effects of genetically engineered effector memory $\mathrm{T}$ cells $\left(\mathrm{T}_{\mathrm{EM}}\right)$ and central memory $\mathrm{T}$ cells $\left(\mathrm{T}_{\mathrm{CM}}\right)$. However only the $\mathrm{T}_{\mathrm{CM}}$ were longlived in vivo and resulted in cures. They also found synergy between CD4 and CD8 TCM in their mouse model. CART cells that were purified CD4 or CD8 were not as effective as a population that was mixed to contain CD4 and CD8 cells. Based on this, they are testing this combined CD4 + CD8 CART approach clinically [16]. A recent patient that responded to this approach has shown on day
10 after CART therapy that $64 \%$ of circulating $\mathrm{T}$ cells are CART-modified.

Anastasia Papadopoulou (Baylor College of Medicine) and colleagues are taking a novel approach towards treating viral infection after HSCT. PBMCs from the HSCT donor are stimulated in vitro with overlapping peptides for ADV, EBV, CMV, BK and HHV6 viruses and cultured/ expanded with IL4/7 in vitro for 10 days. They routinely get cell lines with potent anti-viral specificity but no alloreactivity, based on in vitro testing [17]. So far, 11 patients were treated with these donor-derived anti-viral $\mathrm{T}$ cell lines. Shortly after the infusions, the viremia is seen to dramatically decrease, while anti-viral T-cell reactivity from donor derived $\mathrm{T}$ cells increases (in PBMCs). Some patients have shown complete clearance of detectible virus.

Hiroaki Ikeda (Mie University, Japan) and colleagues have chosen to focus on esophageal cancer, a very difficult clinical setting. They have used genetically engineered $\mathrm{T}$ cells that express TCRs that recognize HLA-A2404 restricted MAGE-A4, found in high density on esophageal cancer [18]. These $\mathrm{T}$ cells mediate potent killing against cell lines in vitro. In adoptive transfer studies in xenograft bearing mice, they can also mediate strong anti-tumor effects. Clinical testing of this approach is underway in a multi-institutional trial. In analyses of initial patients, it is possible to detect these TCR-engineered T cells circulating for many months. These TCR-engineered cells can be removed from the patients PBMCs after therapy, and continue to demonstrate in vitro killing of MAGEA4 bearing tumor cells.

\section{Tumor immune resistance and overcoming It}

Tumors create a local milieu that is highly immunosuppressive. This reflects multiple mechanisms, some known and some still unclear. In addition to immunosuppressive cytokines such as TGF $\beta$, some tumors show extensive upregulation of immunoregulatory molecules such as PD-L1 on tumor cells, or on tumor-associated stromal cells such as DCs. Another resistance mechanism is recruitment of suppressive immune cells such as Tregs and MDSCs into the tumor milieu. In addition to their local effects in the tumor microenvironment, Tregs and MDSCs can also create suppressive effects systemically, at sites such as spleen and tumor-draining lymph nodes. These inhibitory pathways are becoming increasingly viable targets for therapy. However, our mechanistic understanding of how tumors create local and systemic immune suppression is still at an early stage, and much discovery research remains to be done. It is exciting for the field, however, that these pathways have now become an active target for clinical therapy.

Stephen Hodi (Dana Farber Cancer Institute) spoke on clinical development of PD-1/PD-L1 blockade. He reviewed the history of selected agents in the clinic, starting with 
nivolumab (anti-PD-1, Bristol-Myers Squibb, BMS-9365 58), which showed $18-28 \%$ response rates in Phase I, depending on the tumor type. This included a number of long-lasting responses, and also examples of late responses (off study drug) suggestive of an ongoing immune reaction against the tumor. In that study, expression of PD-L1 in the tumor by immunohistochemistry was associated with higher response rate. Additional agents were reviewed, including MK-3475 (Merck) and MPDL3280A (anti-PD-L1, Genentech/Roche), where an association of PD-L1 expression with response rate was again observed. Results of the first combination trial of nivolumab with anti-CTLA-4 (ipilimumab) were recently reported, suggesting enhanced activity of the combination. Future questions for investigation were discussed, including whether synergy can be obtained by simultaneously blocking more than one checkpoint; and which preclinical animal models can best predict clinical outcome of such interventions (a relevant point since PD-1/PD-L1 blockade has worked better in the clinic than might have been predicted from mouse studies).

Holbrook Kohrt (Stanford Cancer Institute) presented an abstract describing biomarkers of response to MPDL 3280A, an engineered antibody directed against PD-L1 (Genentech/Roche). Results were from a Phase I trial of 277 patients with solid tumors. Response rate was 14$30 \%$ depending on tumor type, and patients with PD-L1 expression in the tumor at baseline had a higher rate of response. Baseline expression of PD-L1 was associated with increased infiltration of CD8+ cells and higher expression of IFN- $\gamma$ in the tumor. There were 26 patients with matched on-treatment biopsies available. Responding tumors showed increased CD8+ and Th1 responses over baseline, and a concomitant increase in PD-L1 expression; whereas non-responders showed little inflammatory response in the tumor. These findings suggested that the baseline level of spontaneous $\mathrm{T}$ cell immune response against the tumor might be driving counterregulatory PD-L1 expression, and also became able to drive the response to therapy when PD-L1 was blocked.

Rahul Roychoudhuri (National Cancer Institute) described the role of the transcription factor $\mathrm{BACH} 2$ in differentiation and function of Tregs and effector CD4 cells. Clinically, abnormalities of BACH2 have been associated with multiple forms of autoimmunity. Mice lacking $\mathrm{BACH} 2$ had defective differentiation of Tregs, which was due to the inability to repress competing pro-inflammatory differentiation programs in the CD4 lineage. Thus, $\mathrm{BACH} 2$ is centrally positioned at the choice between tolerance/ Tregs versus inflammation/effector differentiation.

Dmitry Gabrilovich (Wistar Institute) spoke on the fate of myeloid-derived suppressor cells in tumor-bearing hosts. Granulocytic MDSCs progressively increase in the presence of tumors and it has been assumed that they must therefore have improved survival compared to normal granulocytes. In reality, however, granulocytic MDSCs were found to have shortened survival and increased sensitivity to apoptosis. Apoptosis appeared to be mediated via the DR5 receptor and caspase 8 . Engagement of DR5 in mouse models eliminated MDSCs and enhanced antitumor immunotherapy. Human MDSCs likewise appeared more sensitive to apoptosis via TRAIL/DR ligation. Thus, MDSCs may be selectively sensitive to elimination by TRAIL administration.

\section{Vaccines}

The molecular identification of human cancer-specific antigens has allowed the development of antigen-specific immunotherapy. In one approach, autologous antigenspecific T cells are expanded ex vivo and then re-infused into patients. Another approach is through vaccination; that is, the provision of an antigen together with an adjuvant to elicit therapeutic $\mathrm{T}$ cells in vivo. This session discussed cutting edge approaches to cancer vaccines as well as challenges that remain in efforts to generate effective therapeutic immunity.

Howard Kaufman (Rush University) discussed a phase III study testing the clinical activity of GM-CSF encoding Herpes virus based vector (OPTIM Oncovex) in stage IIIb-IV melanoma. By localizing GM-CSF to tumor sites using this approach, the goal is to enable the in situ tumor to function as an endogenous cancer vaccine. The control arm received treatment with GM-CSF only. There was $2.1 \%$ vs $16.3 \%$ durable response rate in the control vs. the Oncovex arm, with complete response $0.7 \%$ vs $10 \%$. 36 month survival was $40.6 \%$ for Oncovex vs $27.8 \%$ for the control arm. These are very encouraging data and hopefully the next step will be combination therapy with checkpoint inhibitors. Another approach to viral vectors was discussed by Dr. Schlom who summarized emerging concepts in vaccine therapy and focused on studies with tumor antigen encoding viral vectors, specifically TRICOM. The PROSTVAC phase II and phase III trial in metastatic prostate cancer showed changes in tumor growth kinetics, resulting in improved overall survival. PANVAC is being tested in metastatic colorectal cancer post surgery. Here as well, some benefit for overall survival (but not DFS) was observed when comparing PANVAC vs standard of care in a single center trial.

Cornelis Melief (Leiden University) discussed outcomes of vaccination with long peptides combined with adjuvants such as CpG or polyIC. In pre-malignant lesions, HPV targeted vaccination led to very promising results with good tumor control, which was dependent on CD8+ T cell immunity. However, the efficacy of this vaccination protocol was much diminished in patients with established $\mathrm{HPV}+$ cancer. Interestingly, vaccination during the myeloid nadir following cis-paltinum improved the outcome. 
Thus, depletion of myeloid cells might enable generation of adaptive immunity in response to vaccination. These very interesting results further support the rationale for combination of immunotherapy with classical treatments in oncology. The discussion of other approaches to enhance immunogenicity included a novel class of adjuvants (Thomas Dubensky, Aduro BioTech) which are based on cyclic di nucleotides able to activate STING; and the role of IL18 which, when secreted from activated NK cells in the presence of IFN alpha, primes DCs for CCL19 secretion (Pawel Kalinski, University of Pittsburgh). This priming of DCs has been observed in mice and also in tumor draining lymph nodes from patients with colorectal cancer. Thus, IL18 acts as bridge between tumor and lymph nodes.

Finally, two presentations focused on challenges to the creation of effective cancer vaccines. Vincent Brichard (GSK) discussed GSK vaccines based on MAGE-3 tumor antigen administered in combination with three adjuvants (AS15) in liposomal formulation. Following positive phase II studies, phase III trials have been developed in lung cancer (MAGRIT) and in melanoma (DERMA) post surgery. The DERMA trial enrolled 1320 patients with two primary endpoints: a) disease free survival in the total population; and b) disease free survival in MAGE3A + tumors. The study did not meet the goal for the 1st endpoint but will continue to assess data for the 2nd endpoint in 2015. A critical point is selection of patients for active immunotherapy; GSK has established a gene signature that is being prospectively validated. Nicholas Restifo (NCI) further underscored the need for patient stratification and outlined three requirements for successful vaccines including: 1 . Choice of target antigen; 2. Matching T cells with targets: 3 . potent $\mathrm{T}$ cell repertoire; and 4. Control of Tumor microenvironment.

\section{Metabolism and immunity}

In recent years, the role of metabolic pathways in regulating both tumor biology and immune responses has gained increasing attention. It has long been recognized that tumors show multiple metabolic abnormalities, including increased dependence on glycolysis (Warburg effect), adaptation to hypoxia, and other changes. In the immune system, factors such as cellular energy balance, nutrient availability, hypoxia and redox state are more than just "housekeeping" functions, they can also be critical determinants of immune-cell activation, differentiation and effector function. Metabolic sensing and control pathways such as mTOR, GCN2 and HIF- $1 \alpha$ are thus emerging as potential therapeutic targets in the immune system, or as checkpoints exploited by the tumor.

Tak Mak (Princess Margaret Hospital, Toronto) summarized the impact of nutrient and metabolic stress in the tumor micro-environment. Tumor cells and tumorinfiltrating immune cells are competing for the same limited resources (including glucose, oxygen, reducing thiols and amino acids), and experience the same environmental metabolic stresses (free radical flux, hypoxia, etc.). Thus, pathways that sense and respond to nutrient-related stress are important for both tumor biology and for local immune responses: examples include mTOR, ER stress (e.g., PERK), autophagy, GCN2, HIF-1 $\alpha$ and redox pathways. Glycolysis is important for tumor cells (Warburg effect), and glycolysis is also required for activation of effector $\mathrm{T}$ cells. In contrast naive $\mathrm{T}$ cells and Tregs may preferentially use fatty-acid metabolism. Thus, regulatory pathways such as mTOR that link glycolysis, energybalance and redox regulation are important for immune function in the tumor microenvironment.

David Munn (Georgia Regents University) discussed the role of metabolic stress as an immunologic checkpoint in $\mathrm{T}$ cells, with a focus on amino acid withdrawal. T cells are sensitive to amino-acid deprivation during activation. Either tryptophan depletion (via IDO expressed in DCs or macrophages), or arginine depletion (via Arginase I expressed in macrophages or MDSCs), can activate the amino-acid sensitive GCN2 pathway. The result is GCN2mediated cell-cycle arrest and anergy in effector T cells; and GCN2-mediated activation in Tregs. mTOR is also potentially a sensor of amino-acid withdrawal stress, although a specific role for mTOR in this regard has not yet been demonstrated. In mouse tumor models, inhibition of IDO with 1-methyl-D-tryptophan (indoximod) or the high-affinity IDO-inhibitor NLG919 (NewLink Genetics) reduced Treg-mediated suppression, and enhanced antitumor responses. Blockade of IDO was synergistic with blockade of the PD-1/PD-ligand pathway. IDO and PD-L1 are both inducible in the tumor microenvironment by $\mathrm{T}$ cell-driven inflammation, as recently reported by Gajewski and colleagues [19], so these pathways may function as inducible counter-regulatory mechanisms to limit antitumor immune responses.

Madhusudhanan Sukumar (National Institutes of Health) described the role of mitochondrial metabolism in regulating CD8+ $\mathrm{T}$ cell memory and self-renewal. Activated $\mathrm{T}$ cells must convert large quantities of nutrients to energy. Different subsets of T cells, and different stages of differentiation, have differing requirements for glycolysis and mitochondrial metabolism. When CD8+ T cells undergo activation and effector differentiation, they become heavily dependent on the glycolytic pathway. Inhibition of glycolysis during activation favors formation of long-lived memory cells, rather than terminal differentiation into short-lived effector cells. Identification and isolation of cells with lower mitochondrial membrane potential (by use of a fluorescent marker dye) allowed enrichment of CD8+ T cells with a more "stem-like" gene-expression profile. Following adoptive transfer, these cells mediated enhanced anti-tumor activity and markedly increased 
memory formation. Lower metabolic activity, with consequent reduced free-radical flux, may help protect longlived cells from free-radical damage and early senescence.

Paolo Rodriguez (Louisiana State University) described therapeutic modulation of $\mathrm{T}$ cell function via arginine metabolism. T cells are sensitive to arginine deprivation, which activates the GCN2 kinase pathway, leading to cellcycle arrest and anergy. Dr. Rodriguez and colleagues have developed a PEG-conjugated form of recombinant human L-arginine (peg-Arg I). In vitro, treatment with peg-Arg I did not affect early activation events in resting $\mathrm{T}$ cells, but it inhibited subsequent cell cycle progression and effector differentiation. In vivo, administration of peg-Arg I created systemic arginine depletion and inhibited $\mathrm{T}$ cell activity. Arginine depletion also was associated with an increase in the number of granulocytic MDSCs in vivo. When tested in disease models (graft-vs-host disease and inflammatory bowel disease), peg-Arg I significantly extended survival of treated mice.

\section{Biomarkers and immunoscore}

The advent of novel but high-cost treatments for metastatic melanoma, such as ipilimumab and anti-PD1 (e.g. nivolumab), has emphasized the need for biomarkers that can be used to predict those patients most likely to respond to therapy. For ipilimumab, we know treatment can improve overall survival but not the surrogate endpoints of overall response or progression-free survival. In addition, specific subgroups of patients with a higher benefit have not been identified. However, as reported at the 2013 European Cancer Congress, about one-fifth of patients who receive ipilimumab are still alive at 10 years [20]. As such, biomarkers to predict these patients with potential long-term benefit from ipilimumab therapy would be very welcome. For anti-PD1 treatment, PD-L1 expression in the tumor might be expected to be a predictive biomarker for response. However, this hypothesis is complicated by that finding that about $20 \%$ of patients who are PD-L1 negative respond to anti-PD1 treatment (see "Hot Topic" summary, below). Finally, there is increased interest in the correlation between the presence of immune infiltrate in the tumor microenvironment and patient outcomes. This infiltrate might predict not only the prognosis (as evidenced for the immunoscore in colorectal cancer but also response to treatment, including chemotherapy as well as immunotherapy [21]. In fact, in a recent published adjuvant trial of patients with breast cancer, the presence of the immune infiltrate correlated with a better outcome only in the triple-negative patients. In the HER2positive population, the presence of infiltrate was associated with a better outcome in patients treated with doxorubicin-based chemotherapy but not in patients receiving docetaxel in addition to doxorubicin-based treatment, suggesting that somehow the addition of the docetaxel might have interfered with some immune-based anti-tumor mechanism in this setting [22].

Mohammed Kashani-Sabet (University of California, San Francisco, CA, USA) and colleagues have found that the Pleckstrin homology domain-interacting protein (PHIP) is a marker and mediator of melanoma metastasis. Overexpression of PHIP is an independent adverse predictor of survival in melanoma patients and a high proportion of melanomas have been reported to harbor an increased PHIP copy number. PHIP-overexpressing tumors include triple-negative (wild-type BRAF, NRAS, and PTEN) and double-negative melanomas (mutant BRAF and wildtype NRAS and PTEN). In 238 patients with melanoma, PHIP copy number was independently predictive of distant metastasis-free survival $(\mathrm{p}=0.03)$ and disease-specific survival $(\mathrm{p}=0.03)$ [23]. Increased PHIP copy number was also predictive of ulceration $(\mathrm{p}=0.04)$. PHIP overexpression may lead to increased glycolytic activity, with lower expression of lactate dehydrogenase (LDH)-5, hypoxia-inducible factor- $1 \alpha$ subunit, and vascular endothelial growth factor, as well as increased micro-vessel density. Increased glycolysis and angiogenesis may be drivers of ulceration and metastasis.

The role of a 12-chemokine gene signature as a marker in the selection of patients for immunotherapy was reported by James J. Mulè (Moffitt Cancer Center, Tampa, FL, USA). This 12-chemokine gene signature predicts the presence of unique, ectopic lymph node-like structures in solid tumors, including metastatic melanomas. A direct correlation between the 12-chemokine gene signature score and the presence of these structures was associated with better overall survival in a small group of melanoma patients [24]. It has been postulated that chemokines produced locally within the microenvironment of the tumor are responsible for the formation of these lymph node-like structures, which may play an important role in eliciting an endogenous anti-tumor immune response, thereby improving patient outcomes. The use of this 12-chemokine gene signature may reveal information on the anti-tumor immune response, potentially leading to improvements in the identification and selection of melanoma patients most likely to respond to immunotherapy.

Margaret K. Callahan (Memorial Sloan-Kettering Cancer Center, New York, NY, USA) reported information on biomarkers from two phase I studies in patients with advanced melanoma treated with the anti-PD1 agent nivolumab alone (trial CA209-003) and in combination with ipilimumab (trial CA209-004). Rapid and durable responses were observed for both nivolumab monotherapy and ipilimumab plus nivolumab combination therapy; these responses persisted after discontinuation of treatment in some patients. Positive PD-L1 staining of pretreatment tumors suggested an increased likelihood of response to nivolumab monotherapy but not ipilimumab 
plus nivolumab combination therapy. Unlike previous reports with ipilimumab monotherapy [25,26], baseline absolute lymphocyte count (ALC) did not predict response to either nivolumab monotherapy or combination therapy. In patients receiving combination therapy, increased numbers of CD4+ and CD8+ T cells expressing activation and proliferation markers were observed while a higher overall response rate correlated with low pre-treatment levels of myeloid-derived suppressor cells (MDSC) in an exploratory single-center subset analysis.

A specific 53-gene panel predictive of non-progression and prolonged survival in two independent cohorts of patients with resectable stage II-III melanoma was identified by Yvonne Saenger using a hypothesis-driven approach (Mount Sinai School of Medicine, New York, NY, USA) and co-workers. These 53 genes have high overlap with a co-expression network identified using unbiased methods using National Center for Biotechnology Information (NCBI) Gene Expression Omnibus (GEO) data. Bayesian network analysis was used to define gene networks surrounding the 53 genes, with key hub genes within defined immune signaling pathways identified as potential key regulators. Th1 processes, $\mathrm{T}$ and $\mathrm{B}$ cell receptor activation, and CD2 were identified as critical modulatory pathways. CD2 staining by immunohistochemistry was also predictive of non-progression and prolonged survival. The 53-gene panel should be prospectively studied in larger trials, while nodes in the Bayesian network could represent potential therapeutic targets.

\section{Combination approaches in cancer}

Historically, neoplastic disease represents a clear example of the importance of combined treatment strategies. In fact, combinations of surgery, radiotherapy and/or chemotherapy have typically represented the best approach for the treatment of various tumors, such as breast, ovarian and head and neck cancer. The development of new compounds, in particular immunomodulatory antibodies and targeted therapies, provide the basis for new combination approaches in the treatment of cancer. The combining, either concurrently or sequentially, of such compounds offers the potential for improved outcomes. Combining these new agents with surgery, radiotherapy and/or chemotherapy may also provide further opportunities in the management of cancer.

Mario Sznol (Yale University School of Medicine, New Haven, CT, USA) discussed various combinations of anticancer immunotherapies based on checkpoint inhibition, in particular a combination of nivolumab plus ipilimumab in a $1: 3$ dose ratio that was associated with a $53 \%$ confirmed objective response rate (three complete and six partial responses) in patients with advanced melanoma [27]. However, there are many unresolved issues regarding dosing and sequencing of combination treatments, e.g. the effects of varying dose ratio on toxicity, whether to use concurrent or sequential therapy, and the possible additional activity of single-agent use after combination therapy. Moreover, optimal management of patients will be complex and will not follow clean protocol-related rules. There must also be an acceptance of increased frequency and severity of adverse events accompanying greater activity. Clinical trial endpoints may also shift from median survival to overall cure rates.

More biomarker data were presented by Laurence Zitvogel (Institute Gustave Roussy, Villejuif, France). Efficacy of ipilimumab was reduced by interleukin (IL)-2R $\beta$ (CD122), IL-2 or IL-15 blockade in mice inoculated with MCA205OVA or MC38OVA ${ }^{\text {dim }}$ tumor cell lines. Moreover, high levels of soluble IL-2 receptor $\alpha$ (sCD25), combined with high LDH-5 levels, predicted resistance to ipilimumab. In 262 patients with metastatic melanoma, high sCD25 was associated with worse survival compared with low sCD25. Soluble CD25 is thought to act as a decoy receptor with immunosuppressive functions. An assessment of biomarkers for response to the engineered anti-PD-L1 antibody MPDL3280A was also reported. In a phase I study in non-small-cell lung cancer, the best overall response rate to $\mathrm{PD}-\mathrm{L} 1$ blockade was seen in patients with more than $10 \%$ tumor immune cells positive for PD-L1. These findings are consistent with the data that pre-treatment PD-L1 expression suggests increased likelihood of response to nivolumab monotherapy.

Stefani Spranger (University of Chicago, Chicago, IL, USA) described how the improved therapeutic efficacy of combination immunotherapy may be associated with re-activation of $\mathrm{T}$ cells directly within the tumor microenvironment. In mice inoculated with B16-SIY tumor cells, tumor control was increased by combined therapy consisting of blockade of CTLA-4 and PD-L1, CTLA-4 and indoleamine-2,3-dioxygenase (IDO) or PD-LI plus IDO versus respective monotherapies. Early $\mathrm{T}$ cell priming was only minimally affected by combination immunotherapies. However, immunotherapy combinations increased the number of CD8+ tumor-infiltrating lymphocytes (TILs) that proliferated and produced IL-2. Combinations also increased the percentage of BrdU $+\mathrm{T}$ cells in the tumor. Altogether, the data indicate that re-activation of tumor residing $\mathrm{T}$ cells mediated tumor control after combination therapy. Further the combination of CTLA-4 and PD-L1 blockade delays tumor outgrowth in subsets of geneticallyinduced melanoma models, with the anti-tumor effect linked to a pre-existing $\mathrm{T}$ cell infiltrate. Thus, cancer immunotherapy approaches targeting negative regulatory immune checkpoints might be preferentially beneficial for patients with a pre-existing $\mathrm{T}$ cell-inflamed tumor microenvironment, with IL-2 production and proliferation of CD8+ TIL potential biomarkers to be investigated in regard to clinical response to immunotherapy. Other potential 
targets in addition to CTLA-4 and PD-L1, including LAG$3,4-1 \mathrm{BB}$, and $\mathrm{OX}-40$, should also be integrated into combination therapy studies.

A novel combination of three immunostimulatory monoclonal antibodies (anti-CD137, anti-OX40 and anti-PD1) was investigated by Aizea Morales-Kastresana (Center for Applied Medical Research [CIMA], University of Navarra, Pamploma, Spain) and colleagues using a transgenic mouse model of multifocal and rapidly progressing hepatocellular carcinoma. The combination extended survival of mice in a CD8-dependent fashion and synergized with adoptive $\mathrm{T}$ cell therapy using activated OVA-specific TCRtransgenic OT-1 and OT-2 lymphocytes. There was an increase in $\mathrm{CD} 3+$ lymphocyte infiltrates in tumor nodules, but not healthy liver tissue. CD8+ TILs were more blastic and showed higher expression of treatment targets, allowing the readministration of the combination. The triple combination also prevented tolerization of transferred activated OT-1 T cells against the well characterized CD8+ $\mathrm{T}$ cell epitope SIINFEKL. TILs from combination-treated mice recognized unknown epitopes on hepatocellular carcinoma-derived cell lines, but not SIINFEKL.

Combination therapies of engineered oncolytic viruses and immunomodulatory antibodies present an attractive therapeutic strategy for clinical exploration in different tumor types. Dmitriy Zamarin (Memorial Sloan-Kettering Cancer Center, New York, NY, USA) reported the use of Newcastle disease virus (NDV), a member of the Paramyxoviridae family, which has been shown to infect a number of avian species. Naturally-occurring attenuated NDV viruses have previously been shown to be effective oncolytic agents against a variety of cancers, including malignant melanoma. Combination therapy with NDV and CTLA-4 blockade leads to rejection of injected and distant B16-F10 tumors and long-term survival in mice. NDV is a strong inducer of type I interferon through the activation of TLR7 and RIG-I pathways and upregulates major histocompatibility complex (MHC) and co-stimulatory molecules on the surface of tumor cells. Moreover, NDV induces upregulation of inducible costimulator (ICOS) on TILs. In combination with anti-CTLA-4, recombinant NDV expressing ICOS ligand (NDV-ICOSL) results in superior therapeutic efficacy compared with wild-type NDV in B16-F10 or CT26 murine tumor models. Moreover, mice cured by combination therapy are protected from further tumor challenge.

New strategies that target the tumor microenvironment to promote local and systemic immune protection were discussed by Yang-Xin Fu (University of Chicago, Chicago, IL, USA). Evidence suggests that radiation is a significant modifier of the tumor microenvironment with specific proimmunogenic effects that facilitate tumor rejection. Optimal radiotherapy-mediated tumor regression is dependent on anti-tumor $\mathrm{T}$ cells with CD8+ T cell depletion reducing therapeutic response to radiotherapy. Moreover, an increase in PD-L1 induces tumor resistance and allows relapse in murine cancer models. Radiation combined with anti-PD-L1 reduces MDSCs through infiltration of cytotoxic T lymphocytes (CTLs) in mice. It is hypothesized that radiotherapy-induced DNA damage leads to excessive DNA fragments, similar to viral infection, which triggers interferon (IFN) release to induce dendritic cell maturation and cross-priming. Similarly, the prevention or slowing of HER $2 /$ neu + tumor growth by the anti-HER2/neu antibody also involves an adaptive immune response. This appears to involve the induction of type I IFNs, with IFNs not being induced in antibody-resistant tumors; in these cases, anti-EGFR antibody armed with IFN $\beta$ is effective. Anti-EGFR-IFN $\beta$ may induce an anti-tumor cytotoxic $\mathrm{T}$ cell-mediated response through an increase of crosspriming. Anti-Her2/neu has also been shown to synergize with IL-21 to rapidly eradicate tumors in mice, with IL-21 amplifying the tumor-specific CD8+ T cell response through polarized tumor-associated macrophages. Local irradiation has also been shown to synergize with IL-21.

\section{Hot topic: is PD-L1 tumor expression necessary?}

This years "hot topic" session was stimulated by the recent clinical testing of anti-PD1 mAb, a new "checkpoint inhibitor", with recent clinical data showing potent activity in patients with refractory melanoma, renal cell cancer as well as non-small cell lung cancer. The controversial issue addressed in this session pertains to the potential need for PD-L1 (the ligand for the PD1 programmed death receptor) to be expressed on a patient's tumor in order for that tumor to respond to anti-PD1 therapy. Drs. Janis Taube (from Johns Hopkins), Joe Grosso [from Bristol-MyerSquibb, (BMS)], and Prim Hegde (from Genentech) each presented slightly different assays that are being used to evaluate clinical specimens for the expression of PD-L1 [28]. These different methods have been used to evaluate separate cancer specimens from patients in distinct clinical trials. For the most part, in clinical testing of single agent blockade using anti-PD1, patients that have tumors that show any clear cut expression of PD-L1 are more likely to respond than patients whose tumors are clearly negative for PD-L1. In the study using the John Hopkins monitoring method, no patients that were PD-L1-negative responded to therapy. In contrast in studies using the BMS or Genetech assays, patients that were PD-L1-negative were less likely to respond than those that were PD-L1+, but approximately $20 \%$ still did respond. The differences seen in these studies might reflect differences in the clinical trials themselves, as well as potential differences in the sensitivity and scoring criteria, to determine PD-L1 positivity vs. negativity. If PD-L1 expression is actually a prerequisite for response to anti-PD1 treatment, then detecting it on a patient's tumor might be a reasonable eligibility criterion 
for future studies. However, if PD-L1 expression on pretreatment samples is not essential for a response to treatment, PD-L1 testing would not be indicated as a treatment eligibility criterion. As the clinical results came from 3 different trials, each evaluated using a distinct assay and criterion for determining PD-L1 status, a suggestion was made to try and resolve this issue. If the samples from all 3 clinical studies could be evaluated for PD-L1 expression, in a blind fashion, by all 3 detection labs (Johns Hopkins, BMS and Genentech), it would clarify distinctions in the detection sensitivity for the 3 separate assays, and provide a greater sample size of patients with which to correlate the potential importance of PD-L1 expression in the response to anti-PD-1 treatment.

\section{Late-breaking abstracts session}

Sylvia Adams (NYU Cancer Institute) described the correlation between histologic analysis of lymphocytic infiltration (a version of the immunoscore) and clinical prognosis in triple-negative breast cancer. Using a large set of outcome data available on patients from two adjuvant-chemotherapy trials (E1199 and E2197), initial biopsy samples were analyzed morphologically by $H \& E$ staining for lymphocyte infiltration in stroma. The degree of stromal lymphocyte infiltration correlated positively with disease-free survival and overall survival following adjuvant chemotherapy. Thus, assessment of lymphocytic infiltration provided additional prognostic information, beyond traditional TNM staging, in a disease for which additional prognostic markers are needed.

Antonio Grimaldi (Instituto Nazionale Tumori Fondazione, Naples) described abscopal responses observed in a group of patients treated with ipilimumab for metastatic melanoma, who subsequently received local radiotherapy for control of progressive disease. The data were from a retrospective analysis of 21 patients receiving either brain or body RT (conventional or stereotactic irradiation) following progression after ipilimumab (median time after ipilimumab treatment was 5 months). Abscopal responses, defined as tumor regression outside of the field of radiation, were observed in $42 \%$ of patients. Patients who had abscopal responses had significantly longer overall survival than patients who did not ( 22 months vs. 8 months). This report thus joins a growing body of evidence suggesting that immunotherapy such as ipilimumab may "prime" the immune system to create systemic anti-tumor responses following local radiotherapy. Conversely, local radiation may trigger immune responses after immunotherapy that would otherwise not spontaneously occur (e.g., even in patients who are actively progressing after immune therapy). It thus becomes relevant to consider the possibility of planned addition of local radiation - either sequentially or concurrently - to ipilimumab therapy, and potentially other forms of immune-activating therapy.

\section{Presidential session}

Interventions such as checkpoint blockade, $\mathrm{T}$ cell adoptive therapy and $\mathrm{T}$ cell vaccines are all becoming clinical realities; but the tumor microenvironment remains a forbidding place for $\mathrm{T}$ cell function. $\mathrm{T}$ cells are often rendered anergic or inhibited in this environment, or they fail to receive the necessary pro-inflammatory signals from the innate immune system to support their activation. Even if they can be successfully pre-activated ex vivo (e.g., TILs and CARs) they may not receive the needed signals to home effectively to the tumor, or they may become suppressed or exhausted when they get there. Thus, strategies to address these various defects and impediments have become highly topical, and of direct translational interest.

Paolo Rodriguez (Louisiana State University) presented data elucidating the effects of Notch-1 in T cells. T cells in the tumor microenvironment are often suppressed or anergic, and a similar state appears to be induced by inhibiting Notch signaling in T cells. Dr. Rodriguez and colleagues showed that Notch-1 and Notch-2 were elevated during normal $\mathrm{T}$ cell activation, and that conditional deletion of Notch-1 and -2 abrogated T cell proliferation and cytokine production. Conversely, transgenic over-expression in T cells of a constitutively-active Notch1 intracellular domain conferred enhanced anti-tumor effect in vivo, and increased expression of survival receptors such as IL-7R. Thus, Notch-1/2 signaling in $T$ cells may act to oppose some of the inhibitory effects of tumors in vivo. It could thus represent a potential therapeutic intervention, or a modification to allow engineered $\mathrm{T}$ cells to be more resistant to the suppressive effects of tumors.

Seng-Ryong Woo (University of Chicago) presented data showing that innate immune cells in the tumor microenvironment sense the presence of growing tumor via the host STING pathway. STING is an intracellular sensor for cytosolic DNA, so activation of this pathway in host DCs requires uptake of tumor DNA. Tracking-dye studies in mouse models revealed that large numbers of host DCs acquired tumor-derived DNA. Xenograft models and other studies suggested that the source of this tumorderived DNA appeared to be from tumor mitochondria. Activation of the STING pathway resulted in local production of IFN $\beta$ and enhanced T cell priming; conversely, highly immunogenic tumors were not rejected in STINGdeficient hosts. Thus, innate sensing of tumor-derived DNA played an important role in priming immune responses against growing tumors.

Ileana Mauldin (University of Virginia, Charlottesville) presented preclinical studies describing the effects of TLRligands on chemokine production by human melanoma cells. Melanoma cell lines expressed multiple Toll-like receptors, but exposure to their various cognate ligands triggered little chemokine production. However, the 
combination of TLR2/6 ligation plus treatment with IFNy resulted in production of the chemokine CXCL10. Expression of TLR2 and TLR6 were confirmed on primary human melanoma samples, as was production of CXCL10 in response to TLR2/6 ligation plus IFN $\gamma$. CXCL10 has been implicated in $\mathrm{T}$ cell recruitment, so this approach could represent a strategy for enhancing recruitment of $\mathrm{T}$ cells into melanoma lesions.

Cara Haymaker (MD Anderson) presented in vitro and in vivo data suggesting that expression of the co-inhibitory receptor BTLA on tumor-infiltrating CD8+ T cells may confer a survival and functional advantage in vivo. Ligation of BTLA by its counter-ligand HVEM is inhibitory for $\mathrm{T}$ cell proliferation. However, in clinical trials of TIL adoptive therapy, patients receiving a higher fraction of BTLA-expressing cells were found to have improved outcome. To follow up this seemingly paradoxical observation, they characterized the BTLA + and BTLA- CD8+ populations in the TIL preparations. The BTLA + cells had a "younger" (less terminally differentiated) gene-expression profile; showed higher proliferation and cytokine production; and were less susceptible to activation-induced cell death (AICD). This latter attribute may be related to the finding that BTLA engagement activated the PI3K-Akt signaling pathway. In vivo tracking of unique CDR3 sequences in the BTLA + and BTLA- sub-populations of clinical TIL preparations showed improved survival and competitive advantage in vivo in the BTLA + fraction. Thus, in the context of highly-activated TIL preparations, the restraining effect and pro-survival signaling of BTLA may confer a functional advantage in vivo.

\section{Updates \\ $\mathrm{NCl}$}

William Merritt (Program Director, Clinical Grants and Contracts Branch/CTEP, National Cancer Institute) gave an update on trends and priorities in funding at the $\mathrm{NCI}$, as well as immunotherapy agents available, and changes in the Early Clinical Trials program. The slides from the presentation are archived on the SITC website and available for review. The number of NCI research grants awarded has been flat since 2011. It is a priority at NCI to maintain the number of investigator-initiated research grants, and a number of cuts in other areas have been made to maintain this level. A list of available CTEP reagents was discussed, and also a discussion of high-priority targets and agents for DCTP/CTEP. It was discussed that significant changes will be made to the NCI Early Clinical Trials Program. The proposed changes include an expectation for molecular characterization of the tumor; an expectation that proposals will address some aspect of critical unanswered questions (whether about the disease itself, biomarkers, or drug combinations); and a multidisciplinary "team science" approach will be required. Operational changes to the program will include a more integrated network, more comprehensive central support (monitoring, datacapture, PK, etc.), accelerated timeline for approval, and enhanced funding for molecular characterization and sample acquisition.

\section{FDA}

Raj Puri (Food and Drug Administration) gave an update on FDA regulatory policy related to cancer immunotherapy. The slides from the presentation are archived on the SITC website and available for review. Dr. Puri reviewed the respective offices within FDA that were responsible for regulation of different oncology products (small-molecules, biologics, cellular and gene therapies, vaccines, etc.). Citations and links were given for several recent "Guidance for Industry" documents relevant to immunotherapy, including guidance on trial design, expanded-access programs, co-development of agents for use as combinations, and other areas. Also discussed were "breakthrough therapy" designation; fast-track designation; and acceleratedapproval process. Links were provided to FDA websites, documents and contact information.

\section{The Cancer Immunotherapy Trials Network (CITN)}

The CITN is an NCI-supported consortium, led by PI, Dr. Martin (Mac) Cheever (Fred Hutchinson Cancer Research Center), that brings together cancer immunologists from 29 universities and cancer centers in North America to design and conduct early phase trials for patients with cancer, to provide the infrastructure essential for collaboration, and to gain access to top-ranked agents not broadly available for testing. Since its establishment in 2011, several clinical trials have been formulated, with some already activated and others nearing activation. These include clinical trials of IL-15, IL-15/IL15R $\alpha$-fusion protein, anti-PD1, anti-CD40 mAb, IL-7, IDO-inhibitor, Flt3 ligand. Of the top 11 agents identified in the initial NCI-Immunotherapy agents 2007 workshops [29], 6 are in CITN trials, 3 are no longer being manufactured, 1 is not yet available, and one is not being pursued by CITN. This record indicates the success of the CITN in its initial mission, and the importance of making these promising experimental agents available to the academic research community, through partnership with the NCI and Industry, as facilitated by this collaborative CITN network.

\section{Conclusion}

Certain mAbs and their derivatives can augment ADCC by specific interactions with molecules expressed on tumor cell surfaces. For example, in addition to the recently approved glycoengineered CD20 antibody obinutuzumab, anti-CD137 enhances the efficacy of tumor-reactive mAbs, such as rituximab, while anti-GD2 has shown activity in neuroblastoma when combined with IL-2 and GM-CSF. 
Adoptive cellular immunotherapy has also made much progress over the past year, in particular the preclinical and clinical development of chimeric antigen receptor modified $\mathrm{T}$ cells (CART). Another area of focus for research has been to identify biomarkers predictive of response to immunotherapy. These include PD-L1 tumor expression for anti-PD1 therapy, although this association is not yet clear. High levels of sCD25 combined with high LDH-5 levels predict resistance to ipilimumab and represent a potential biomarker for CLTA-4 blockade. Gene signatures may also represent a feasible approach for selecting patients for immunotherapy. The importance of biomarkers is also apparent in the context of various combination therapy approaches. Combined anti-PD1 and anti-CTLA-4 therapy appears to be promising, as do various other combinations of immunomodulatory mAbs, such as anti-CD137, anti-LAG-3, anti-4-1BB, anti-OX40), as well as immunotherapies combined with vaccines or radiotherapy.

It was clear from this meeting that immunotherapy increasingly offers the potential to improve outcomes for patients with advanced cancers of a wide variety of types. Research activity in this field continues to progress and our understanding of the tumor microenvironment, innate and adoptive immune responses, the activity of various immunotherapeutic modalities, and how to identify patients most likely to benefit from these treatments, is expanding at a rapid rate. This increasing momentum should hopefully enable immunotherapy to become a recognized cornerstone of cancer treatment applicable to many more cancer types and of benefit to many more patients in the future than was ever possible in the past.

\section{Competing interests}

P.A.A. has/had a consultant/advisory role for Bristol Myers Squibb, Roche-Genentech, Merck Sharp \& Dohme, Glaxo SmithKline, Ventana, and Novartis. He received research funds from Bristol Myers Squibb, Roche-Genentech and Ventana. D.H.M. has intellectual property interests in the therapeutic use of IDO inhibitors and is a consultant to NewLink Genetics, Inc. A.K.P. has grant support from Hoffman La Roche for cancer vaccine development. P.M.S. has declared no conflict of interest.

\section{Authors' contributions}

The authors PAA, DHM, AKP and PMS equally contributed to the writing of the manuscript. All authors read and approved the final manuscript.

\section{Acknowledgement}

The authors thank all staff of SITC for their help in organizing the 2013 meeting, and especially Amy Russart, Andrea Rindo and Tara Withington for their help in coordinating the compilation of this annual report.

\footnotetext{
Author details

'Unit of Melanoma, Cancer Immunotherapy and Innovative Therapy, Istituto Nazionale Tumori Fondazione "G. Pascale", Naples, Italy. ${ }^{2}$ Cancer Immunology, Inflammation and Tolerance Program, GRU Cancer Center, Georgia Regents University, Augusta, GA 30912, USA. ${ }^{3}$ Baylor Institute for Immunology Research, Dallas, TX 75204, USA. ${ }^{4}$ Ichan School of Medicine at Mount Sinai, New York, NY 10029, USA. ${ }^{5}$ Departments of Pediatrics and Human Oncology, University of Wisconsin, 4159 MACC Fund UW Childhood Cancer Research Wing Wisconsin Institute for Medical Research, 1111 Highland Avenue, Madison, WI 53705, USA.
}

Received: 3 April 2014 Accepted: 14 April 2014

Published: 15 May 2014

\section{References}

1. Couzin-Frankel J: Breakthrough of the year 2013. Cancer Immunotherapy Sci 2013, 342(6165):1432-1433.

2. Naik S, Bouladoux N, Wilhelm C, Molloy MJ, Salcedo R, Kastenmuller W, Deming C, Quinones M, Koo L, Conlan S, Spencer S, Hall JA, Dzutsev A, Kong H, Campbell DJ, Trinchieri G, Segre JA, Belkaid Y: Compartmentalized control of skin immunity by resident commensals. Science 2012, 337(6098):1115-1119.

3. lida N, Dzutsev A, Stewart CA, Smith L, Bouladoux N, Weingarten RA, Molina DA, Salcedo R, Back T, Cramer S, Dai RM, Kiu H, Cardone M, Naik S, Patri AK, Wang E, Marincola FM, Frank KM, Belkaid Y, Trinchieri G, Goldszmid RS: Commensal bacteria control cancer response to therapy by modulating the tumor microenvironment. Science 2013, 342(6161):967-970.

4. Gross G, Gorochov G, Waks T, Eshhar Z: Generation of effector T cells expressing chimeric $T$ cell receptor with antibody type-specificity. Transplant Proc 1989, 21(1 Pt 1):127-130.

5. Porter DL, Levine BL, Kalos M, Bagg A, June CH: Chimeric antigen receptor-modified T cells in chronic lymphoid leukemia. $N$ Engl I Med 2011, 365(8):725-733.

6. Grupp SA, Kalos M, Barrett D, Aplenc R, Porter DL, Rheingold SR, Teachey DT, Chew A, Hauck B, Wright JF, Milone MC, Levine BL, June CH: Chimeric antigen receptor-modified T cells for acute lymphoid leukemia. N Eng/ J Med 2013, 368(16):1509-1518.

7. Ghiringhelli F, Apetoh L: The interplay between the immune system and chemotherapy: emerging methods for optimizing therapy. Expert Rev Clin Immunol 2014, 10(1):19-30.

8. Chiba S, Baghdadi M, Akiba H, Yoshiyama H, Kinoshita I, Dosaka-Akita H, Fujioka Y, Ohba Y, Gorman JV, Colgan JD, Hirashima M, Uede T, Takaoka A, Yagita H, Jinushi M: Tumor-infiltrating DCs suppress nucleic acid-mediated innate immune responses through interactions between the receptor TIM-3 and the alarmin HMGB1. Nat Immunol 2012, 13(9):832-842.

9. DeNardo DG, Brennan DJ, Rexhepaj E, Ruffell B, Shiao SL, Madden SF, Gallagher WM, Wadhwani N, Keil SD, Junaid SA, Rugo HS, Hwang ES, Jirstrom K, West BL, Coussens LM: Leukocyte complexity predicts breast cancer survival and functionally regulates response to chemotherapy. Cancer Discov 2011, 1(1):54-67.

10. Taylor C, Hershman D, Shah N, Suciu-Foca N, Petrylak DP, Taub R, Vahdat L, Cheng B, Pegram M, Knutson KL, Clynes R: Augmented HER-2 specific immunity during treatment with trastuzumab and chemotherapy. Clin Cancer Res 2007, 13(17):5133-5143.

11. Kohrt HE, Houot R, Weiskopf K, Goldstein MJ, Scheeren F, Czerwinski D, Colevas AD, Weng WK, Clarke MF, Carlson RW, Stockdale FE, Mollick JA, Chen L, Levy R: Stimulation of natural killer cells with a CD137-specific antibody enhances trastuzumab efficacy in xenotransplant models of breast cancer. J Clin Invest 2012, 122(3):1066-1075.

12. Marabelle A, Kohrt H, Levy R: Intratumoral anti-CTLA-4 therapy: enhancing efficacy while avoiding toxicity. Clin Cancer Res 2013, 19(19):5261-5263.

13. Herter $S$, Herting F, Mundigl O, Waldhauer I, Weinzierl T, Fauti T, Muth $G$, Ziegler-Landesberger D, Van Puijenbroek E, Lang S, Duong MN, Reslan L, Gerdes CA, Friess T, Baer U, Burtscher H, Weidner M, Dumontet C, Umana P, Niederfellner G, Bacac M, Klein C: Preclinical activity of the type II CD20 antibody GA101 (obinutuzumab) compared with rituximab and ofatumumab in vitro and in xenograft models. Mol Cancer Ther 2013, 12(10):2031-2042.

14. Yu AL, Gilman AL, Ozkaynak MF, London WB, Kreissman SG, Chen HX, Smith M, Anderson B, Villablanca JG, Matthay KK, Shimada H, Grupp SA, Seeger R, Reynolds CP, Buxton A, Reisfeld RA, Gillies SD, Cohn SL, Maris JM, Sondel PM, G. Children's Oncology: Anti-GD2 antibody with GM-CSF, interleukin-2, and isotretinoin for neuroblastoma. N Eng/ J Med 2010, 363(14):1324-1334.

15. Delgado DC, Hank JA, Kolesar J, Lorentzen D, Gan J, Seo S, Kim K, Shusterman S, Gillies SD, Reisfeld RA, Yang R, Gadbaw B, DeSantes KB, London WB, Seeger RC, Maris JM, Sondel PM: Genotypes of NK cell KIR receptors, their ligands, and Fcgamma receptors in the response of neuroblastoma patients to Hu14.18-IL2 immunotherapy. Cancer Res 2010, 70(23):9554-9561.

16. Jensen MC, Riddell SR: Design and implementation of adoptive therapy with chimeric antigen receptor-modified T cells. Immunol Rev 2014, 257(1):127-144. 
17. Kaloyannidis P, Leen AM, Papadopoulou A: T-cell therapy: a powerful too for the management of viral infections and relapse post hematopoietic stem cell transplantation. Expert Rev Hematol 2012, 5(5):471-473.

18. Ikeda H, Shiku H: Antigen-receptor gene-modified T cells for treatment of glioma. Adv Exp Med Biol 2012, 746:202-215.

19. Spranger S, Spaapen RM, Zha Y, Williams J, Meng Y, Ha TT, Gajewski TF: Up-Regulation of PD-L1, IDO, and Tregs in the melanoma tumor microenvironment is driven by CD8+ T cells. Sci Transl Med 2013, 5:200ra116.

20. Schadendorf D, Hodi FS, Robert C: Pooled analysis of long-term survival data from phase II and phase III trials of ipilimumab in metastatic or locally advanced, unresectable melanoma, in European Cancer Congress 2013 (ECCO-ESMO-ESTRO). Amsterdam, Netherlands: 2013.

21. Galon J, Costes A, Sanchez-Cabo F, Kirilovsky A, Mlecnik B, Lagorce-Pages C, Tosolini M, Camus M, Berger A, Wind P, Zinzindohoue F, Bruneval P, Cugnenc PH, Trajanoski Z, Fridman WH, Pages F: Type, density, and location of immune cells within human colorectal tumors predict clinical outcome. Science 2006, 313(5795):1960-1964.

22. Loi $S$, Sirtaine N, Piette F, Salgado R, Viale G, Van Eenoo F, Rouas G, Francis P, Crown JP, Hitre E, de Azambuja E, Quinaux E, Di Leo A, Michiels S, Piccart MJ, Sotiriou C: Prognostic and predictive value of tumor-infiltrating lymphocytes in a phase III randomized adjuvant breast cancer trial in node-positive breast cancer comparing the addition of docetaxel to doxorubicin with doxorubicin-based chemotherapy: BIG 02-98. J Clin Oncol 2013, 31(7):860-867.

23. Bezrookove V, De Semir D, Nosrati M, Tong S, Wu C, Thummala S, Dar AA, Leong SP, Cleaver JE, Sagebiel RW, Miller JR 3rd, Kashani-Sabet M: Prognostic impact of PHIP copy number in melanoma: linkage to ulceration. J Invest Dermatol 2014, 134(3):783-790.

24. Messina JL, Fenstermacher DA, Eschrich S, Qu X, Berglund AE, Lloyd MC, Schell MJ, Sondak VK, Weber JS, Mule JJ: 12-Chemokine gene signature identifies lymph node-like structures in melanoma: potential for patient selection for immunotherapy? Sci Rep 2012, 2:765.

25. Ku GY, Yuan J, Page DB, Schroeder SE, Panageas KS, Carvajal RD, Chapman PB, Schwartz GK, Allison JP, Wolchok JD: Single-institution experience with ipilimumab in advanced melanoma patients in the compassionate use setting: lymphocyte count after 2 doses correlates with survival. Cancer 2010, 116(7):1767-1775.

26. Delyon J, Mateus C, Lefeuvre D, Lanoy E, Zitvogel L, Chaput N, Roy S, Eggermont AM, Routier E, Robert C: Experience in daily practice with ipilimumab for the treatment of patients with metastatic melanoma: an early increase in lymphocyte and eosinophil counts is associated with improved survival. Ann Oncol 2013, 24(6):1697-1703.

27. Wolchok JD, Kluger H, Callahan MK, Postow MA, Rizvi NA, Lesokhin AM, Segal NH, Ariyan CE, Gordon RA, Reed K, Burke MM, Caldwell A, Kronenberg SA, Agunwamba BU, Zhang X, Lowy I, Inzunza HD, Feely W, Horak CE, Hong Q, Korman AJ, Wigginton JM, Gupta A, Sznol M: Nivolumab plus ipilimumab in advanced melanoma. N Eng/J Med 2013, 369(2):122-133.

28. Lipson EJ, Sharfman WH, Drake CG, Wollner I, Taube JM, Anders RA, Xu H, Yao S, Pons A, Chen L, Pardoll DM, Brahmer JR, Topalian SL: Durable cancer regression off-treatment and effective reinduction therapy with an anti-PD-1 antibody. Clin Cancer Res 2013, 19(2):462-468.

29. Cheever MA: Twelve immunotherapy drugs that could cure cancers. Immunol Rev 2008, 222:357-368.

doi:10.1186/2051-1426-2-15

Cite this article as: Ascierto et al: Highlights and summary of the 28th annual meeting of the Society for Immunotherapy of Cancer. Journal for Immunotherapy of Cancer 2014 2:15.

\section{Submit your next manuscript to BioMed Central and take full advantage of:}

- Convenient online submission

- Thorough peer review

- No space constraints or color figure charges

- Immediate publication on acceptance

- Inclusion in PubMed, CAS, Scopus and Google Scholar

- Research which is freely available for redistribution

Submit your manuscript at www.biomedcentral.com/submit 